\title{
Random vibration of laminated plates modeled within a high-order shear deformation theory
}

\author{
Gabriel Cederbaum \\ Department of Solid Mechanics, Materials and Structures, Tel-Aviv University, Ramat-Aviv, Israel \\ Liviu Librescu ${ }^{\text {a) }}$ \\ Department of Engineering Science and Mechanics, Virginia Polytechnic Institute and State University, \\ Blacksburg, Virginia 24061 \\ Isaac Elishakoff \\ Department of Aeronautical Engineering, Technion-Israel Institute of Technology, Haifa, Israel
}

(Received 25 August 1987; accepted for publication 22 April 1988)

\begin{abstract}
This article deals with the dynamic response of simply supported, symmetric cross-ply laminates to stationary random load. The theory of laminated plates used here takes into account transverse shear flexibility, transverse normal stress, and rotary inertia effects for orthotropic and transversely isotropic laminates. Two cases of random pressure fields are considered in this analysis. In the first case, the random pressure field is modeled as a point load, random in time, with constant spectral density (ideal white noise), while in the second case, it is modeled as a turbulent boundary layer pressure fluctuation. The analysis presented herein, as well as the obtained response characteristics expressed in terms of mean squares, may be useful in the reliability computation of composite structures subjected to random pressure fields.
\end{abstract}

PACS numbers: 43.40.Dx, 43.40.At

\section{INTRODUCTION}

A great deal of interest in the substantiation of the theory of laminated composite plates and shells has been manifested in the literature. This interest was stimulated by the advent of new composite materials and by their increased use in plate and shell construction systems. The special properties exhibited by these new materials, such as, e.g., high degrees of anisotropy and weak rigidities in transverse shear, require new methods of analysis for the associated plate and shell-type structures that should be based upon discarding the classical Kirchhoff assumptions.

Such refined high-order shear deformation theories (HSDT) incorporating transverse shear deformation and transverse normal effects accounting for the higher-order effects and fulfilling the static conditions on the external bounding planes (surfaces) of the plate (shells) will contribute to a better description of their static and dynamic response characteristics. One of the aims of this article is to model the bending theory of composite plates by incorporating the previously mentioned effects. This theory could be viewed as the laminated counterpart of the one developed in Refs. 1 and 2 for the case of a single-layered plate. The dynamic response to low-velocity impact of single-layered plates using the above theory was discussed in Ref. 3. The random response of laminated composite plates by taking into account the transverse shear deformation effect was investigated in very few works ${ }^{4-6}$ that are based on the firstorder transverse shear deformation theory (FSDT).,8

This article deals with the response of simply supported rectangular laminated plates to random loads. The plate is

\footnotetext{
a) On leave from the Tel-Aviv University, Ramat-Aviv, Israel.
}

considered composed of orthotropic layers, symmetrically disposed with respect to its midplane. Two cases of stationary timewise random loads are considered in this study: (a) the case of a point load, random in time, characterized by a constant spectral density (ideal white noise); and (b) the case of pressure fluctuations in a turbulent boundary layer.

The structural response characteristics obtained in this framework are compared with their counterparts obtained within the classical plate theory (CPT) and within the FSDT.

\section{REFINED HIGHER-ORDER THEORY}

In the following analysis, the distribution of the displacement field across the plate thickness is considered as

$$
\begin{aligned}
& U_{1}=z \psi_{x}-\left(4 / 3 h^{2}\right) z^{3}\left(\psi_{x}+W_{, x}\right), \\
& U_{2}=z \psi_{y}-\left(4 / 3 h^{2}\right) z^{3}\left(\psi_{y}+W_{, y}\right), \quad U_{3}=W,
\end{aligned}
$$

where $U_{1}, U_{2}$, and $U_{3}$ are the components of the 3-D displacement vector in the directions $x, y$, and $z$, respectively; $\psi_{x}$ and $\psi_{y}$ denote the rotations of the normals to the midplane about the $y$ and $x$ axes, respectively; and ( ), denotes the partial derivative with respect to the indicated coordinate. This displacement field was used by Ambartsumian ${ }^{9}$ and Reddy. ${ }^{10}$

The representation (1) of the displacement field results in a parabolic distribution of transverse shear strains throughout the plate thickness and in the fulfillment of the condition of zero in-plane loads on the bounding planes of the plate. In such a way the necessity of introducing a transverse shear correction factor, like in the framework of FSDT, is removed. The distribution of the strain components, following from Eq. (1), is 


$$
\begin{aligned}
& \epsilon_{1} \equiv \epsilon_{11}=z \psi_{x, x}-\left(4 / 3 h^{2}\right) z^{3}\left(\psi_{x, x}+W_{, x x}\right), \\
& \epsilon_{2} \equiv \epsilon_{22}=z \psi_{y, y}-\left(4 / 3 h^{2}\right) z^{3}\left(\psi_{y, y}+W_{, y y}\right), \quad \epsilon_{3} \equiv \epsilon_{33}=0 \\
& \epsilon_{4} \equiv 2 \epsilon_{23}=\psi_{y}+W_{, y}-\left(4 / h^{2}\right) z^{2}\left(\psi_{y}+W_{, y}\right) \\
& \epsilon_{5} \equiv 2 \epsilon_{13}=\psi_{x}+W_{, x}-\left(4 / h^{2}\right) z^{2}\left(\psi_{x}+W_{, x}\right) \\
& \epsilon_{6} \equiv 2 \epsilon_{12}= z\left(\psi_{x, y}+\psi_{y, x}\right)-\left(4 / 3 h^{2}\right) \\
& \times z^{3}\left(\psi_{x, y}+\psi_{y, x}+2 W_{, x y}\right)
\end{aligned}
$$

For an orthotropic material, in which the elastic axes of the layer coincide with the geometrical ones, the pertinent constitutive equations may be expressed as

$$
\begin{aligned}
\left\{\begin{array}{l}
\sigma_{1} \\
\sigma_{2} \\
\sigma_{4} \\
\sigma_{5} \\
\sigma_{6}
\end{array}\right\}= & {\left[\begin{array}{lllll}
Q_{11} & Q_{12} & & & \\
Q_{12} & Q_{22} & & 0 & \\
& & Q_{44} & & \\
0 & & Q_{55} & \\
& & & & Q_{66}
\end{array}\right]\left\{\begin{array}{l}
\epsilon_{1} \\
\epsilon_{2} \\
\epsilon_{4} \\
\epsilon_{5} \\
\epsilon_{6}
\end{array}\right\} } \\
& +\sigma_{3}\left\{\begin{array}{c}
R_{11} \\
R_{22} \\
0 \\
0 \\
0
\end{array}\right\},
\end{aligned}
$$

where

$$
\begin{aligned}
& Q_{11}=E_{1} / \Omega, \quad Q_{22}=E_{2} / \Omega, \\
& Q_{12}=v_{12} E_{2} / \Omega, \quad \Omega \equiv 1-v_{12} v_{21}, \\
& Q_{44}=G_{23}, \quad Q_{55}=G_{13}, \quad Q_{66}=G_{12}, \\
& R_{11}=\frac{E_{1}}{E_{3}} \frac{v_{31}+v_{21} v_{32}}{\Omega}, \quad R_{22}=\frac{E_{2}}{E_{3}} \frac{v_{32}+v_{12} v_{31}}{\Omega},
\end{aligned}
$$

with $R_{11}$ and $R_{22}$ the reduced elastic constants.

The distribution of the transverse normal stress $\sigma_{3}$ can be obtained by integration across the segment $[0, z)$ of the equation of motion of the 3-D elasticity theory, written in the absence of body forces as

$$
\sigma_{i 3, i}=\rho \ddot{U}_{3}, \quad i=1,2,3,
$$

where $\rho$ denotes the mass density and the dots denote the time derivative. This yields

$$
\begin{aligned}
\sigma_{3}= & z\left[\rho \ddot{U}_{3}-Q_{55}\left(\psi_{x, x}+W_{, x x}\right)-Q_{44}\left(\psi_{y, y}+W_{, y y}\right)\right] \\
& -\left(4 / 3 H^{2}\right) z^{3}\left(Q_{55} W_{, x x}+Q_{44} W_{, y y}\right) .
\end{aligned}
$$

The definitions of the stress resultants $L_{i j}$ and the stress couples $L_{i 3}(i, j=1,2)$, intervening in the bending equations of motion of the theory of plates, are given by

$$
L_{i j}=\sum_{k=1}^{N} \int_{z_{k}}^{z_{k+1}} z \sigma_{i j}^{(k)} d z, \quad L_{i 3}=\sum_{k=1}^{N} \int_{z_{K}}^{z_{k+1}} z \sigma_{i 3}^{(k)} d z,
$$

where $N$ denotes the total number of layers.

The equations of motion, necessary for the solution of this problem and expressed in terms of the 2-D quantities defined by Eq. (7), are

$$
L_{1 j, j}-L_{13}=f_{1}, \quad L_{2 i, i}-L_{23}=f_{2}, \quad L_{i 3, i}+P_{3}=f_{3},
$$

where $P_{3}$ denotes the transverse external load, while

$$
f_{i}=\int_{-h / 2}^{h / 2} \rho z \ddot{U}_{i} d z, \quad f_{3}=\int_{-h / 2}^{h / 2} \rho z \ddot{U}_{3} d z
$$

denote rotatory and transversal inertia terms, respectively.

Equations (8) may be obtained through integration across the plate thickness of the equations of motion of the 3D elasticity theory. Finally, the governing equations associated with the bending theory are obtained by replacing in Eqs. (8) the stress resultants and stress couples expressed in terms of the unknowns $\psi_{x}, \psi_{y}$, and $W$. Using in addition the proportional damping model ( $C$ is the damping factor), the governing equations are written as follows:

$$
\begin{aligned}
\left(D_{11}\right. & \left.-\frac{4}{3 h^{2}} F_{11}\right) \psi_{x, x x}+\left(D_{66}-\frac{4}{3 h^{2}} F_{66}\right) \psi_{x, y y}+\left(D_{12}-\frac{4}{3 h^{2}} F_{12}\right) \psi_{y, x y}+\left(D_{66}-\frac{4}{3 h^{2}} F_{66}\right) \psi_{y, x y} \\
& -\frac{4}{3 h^{2}} F_{11} W_{, x x x}-\frac{4}{3 h^{2}}\left(F_{12}+F_{66}\right) W_{, x y y}-\left(A_{55}-\frac{4}{h^{2}} D_{55}\right)\left(\psi_{x}+W_{, x}\right) \\
& -\left(\bar{D}_{55}-\frac{4}{3 h^{2}} \bar{F}_{55}\right)\left(W_{, x x x}+\psi_{x, x x}\right)-\left(\bar{D}_{44}-\frac{4}{3 h^{2}} \bar{F}_{44}\right)\left(W_{, x y y}+\psi_{y, x y}\right) \\
& =\left(I_{3}-\frac{4}{3 h^{2}} I_{5}\right)\left(\ddot{\psi}_{x}+C \dot{\psi}_{x}\right)-\left(\frac{4}{3 h^{2}} I_{5}+\bar{I}_{31}\right)\left(\ddot{W}_{, x}+C \dot{W}_{, x}\right) \\
\left(D_{22}\right. & \left.-\frac{4}{3 h^{2}} F_{22}\right) \psi_{y, y y}+\left(D_{66}-\frac{4}{3 h^{2}} F_{66}\right) \psi_{y, x x}+\left(D_{21}-\frac{4}{3 h^{2}} F_{21}\right) \psi_{x, y x}+\left(D_{66}-\frac{4}{3 h^{2}} F_{66}\right) \psi_{x, y x} \\
& -\frac{4}{3 h^{2}} F_{22} W_{, y y y}-\frac{4}{3 h^{2}}\left(F_{21}+F_{66}\right) W_{, y x x}-\left(A_{44}-\frac{4}{h^{2}} D_{44}\right)\left(\psi_{y}+W_{, y}\right) \\
& -\left(\bar{D}_{44}-\frac{4}{3 h^{2}} \bar{F}_{44}\right)\left(W_{, y y y}+\psi_{y, y y}\right)-\left(\bar{D}_{55}-\frac{4}{3 h^{2}} \bar{F}_{55}\right)\left(W_{, y x x}+\psi_{x, y x}\right) \\
& =\left(I_{3}-\frac{4}{3 h^{2}} I_{5}\right)\left(\ddot{\psi}_{y}+C \dot{\psi}_{y}\right)-\left(\frac{4}{3 h^{2}} I_{5}+\bar{I}_{32}\right)\left(\ddot{W}_{, x}+C \dot{W}_{, y}\right) \\
\left(A_{55}\right. & \left.-\frac{4}{h^{2}} D_{55}\right)\left(W_{, x x}+\psi_{x, x}\right)+\left(A_{44}-\frac{4}{h^{2}} D_{44}\right)\left(W_{, y y}+\psi_{y, y}\right)+P_{3}=I_{1}(\ddot{W}+C \dot{W}) .
\end{aligned}
$$


In (9) the rigidities and inertia terms are defined as

$$
\begin{aligned}
& \left(D_{i j}, F_{i j}\right)=\int_{-h / 2}^{h / 2} Q_{i j}\left(z^{2}, z^{4}\right) d z \quad(i j=1,2), \\
& \left(D_{i i}, F_{i i}\right)=\int_{-h / 2}^{h / 2} Q_{i i}\left(z^{2}, z^{4}\right) d z \quad(i=6), \\
& \left(A_{i i}, D_{i i}\right)=\int_{-h / 2}^{h / 2} Q_{i i}\left(1, z^{2}\right) d z \quad(i=4,5), \\
& \left(\bar{D}_{44}, \bar{F}_{44}\right)=\int_{-h / 2}^{h / 2} Q_{44} R_{11}\left(z^{2}, z^{4}\right) d z \\
& \left(\bar{D}_{55}, \bar{F}_{55}\right)=\int_{-h / 2}^{h / 2} Q_{55} R_{22}\left(z^{2}, z^{4}\right) d z \\
& \left(I_{1}, I_{3}, I_{5}\right)=\int_{-h / 2}^{h / 2} \rho\left(1, z^{2}, z^{4}\right) d z \\
& \bar{I}_{3 i}=\int_{-h / 2}^{h / 2} \rho R_{i i} z^{2} d z \quad(i=1,2) .
\end{aligned}
$$

\section{DYNAMIC RESPONSE}

Equations (9) constitute a system of partial differential equations of the sixth order. For the case of simply supported rectangular panels $(a \times b)$, we write the boundary conditions as

$$
\begin{aligned}
& W=\psi_{y}=L_{11}=0, \quad \text { at } x=0, a, \\
& W=\psi_{x}=L_{22}=0, \quad \text { at } y=0, b .
\end{aligned}
$$

The solution functions are then represented in a form that fulfills exactly the boundary conditions:

$$
\begin{aligned}
& \psi_{x}(x, y, t)=\sum_{m, n} \hat{X}_{m n} \cos \alpha x \sin \beta y T_{m n}(t) \equiv \sum_{m, n} X_{m n} T_{m n}, \\
& \psi_{y}(x, y, t)=\sum_{m, n} \hat{Y}_{m n} \sin \alpha x \cos \beta y T_{m n}(t) \equiv \sum_{m, n} Y_{m n} T_{m n}, \\
& W(x, y, t)=\sum_{m, n} \hat{W}_{m n} \sin \alpha x \sin \beta y T_{m n}(t) \equiv \sum_{m, n} W_{m n} T_{m n},
\end{aligned}
$$

where $\alpha=m \pi / a, \beta=n \pi / b, \hat{X}_{m n}, \hat{Y}_{m n}, \hat{W}_{m n}$ are the coefficients of the natural mode shapes associated with the free vibration problem, while $T_{m n}(t)$ denote the generalized coordinates. The transverse loading function is given by

$$
P_{3}=P_{3}(x, y, t)=\sum_{m, n} q_{m n} \sin \alpha x \sin \beta y F_{m n}(t),
$$

where $q_{m n}$ are the Fourier coefficients. For the free undamped vibration problem, $F_{m n}(t) \equiv 0, C \equiv 0$, and $T_{m n}(t)=e^{i \omega_{m n} t}(i=\sqrt{-1})$; and using (12) in the governing equations (9), we obtain the eigenvalue problem in the form

$$
\left[[K]_{m n}-\omega_{m n}^{2}[M]_{m n}\right]\{\Delta\}_{m n}=\{0\}
$$

where

$$
\{\Delta\}_{m n}^{T}=\left\{\hat{X}_{m n}, \hat{Y}_{m n}, \hat{W}_{m n}\right\}
$$

The elements of the $3 \times 3[K]$ and $[M]$ matrices are

$$
\begin{aligned}
& k_{11}=D_{11} \alpha^{2}+D_{66} \beta^{2}+A_{55}-\left(4 / h^{2}\right) D_{55}-\left(4 / 3 h^{2}\right)\left(F_{11} \alpha^{2}+F_{66} \beta^{2}\right)-\left[\bar{D}_{55}-\left(4 / 3 h^{2}\right) \bar{F}_{55}\right] \alpha^{2}, \\
& k_{12}=\left\{D_{12}+D_{66}-\left(4 / 3 h^{2}\right)\left(F_{12}-F_{66}\right)-\left[\bar{D}_{44}-\left(4 / 3 h^{2}\right) \bar{F}_{44}\right]\right\} \alpha \beta, \\
& k_{13}=\left(A_{55}-\frac{4}{h^{2}} D_{55}\right) \alpha-\frac{4}{3 h^{2}}\left[F_{11} \alpha^{3}-\left(F_{12}+2 F_{66}\right) \alpha \beta^{2}\right]-\left(\bar{D}_{55}-\frac{4}{3 h^{2}} \bar{F}_{55}\right) \alpha^{3}-\left(\bar{D}_{44}-\frac{4}{3 h^{2}} \bar{F}_{44}\right) \alpha \beta^{2}, \\
& k_{21}=\left\{D_{21}+D_{66}-\left(4 / 3 h^{2}\right)\left(F_{21}+F_{66}\right)-\left[\bar{D}_{55}-\left(4 / 3 h^{2}\right) \bar{F}_{55}\right]\right\} \alpha \beta, \\
& k_{22}=D_{22} \beta^{2}+D_{66} \alpha^{2}+A_{44}-\left(4 / h^{2}\right) D_{44}-\left(4 / 3 h^{2}\right)\left(F_{22} \beta^{2}+F_{66} \alpha^{2}\right)-\left[\bar{D}_{44}-\left(4 / 3 h^{2}\right) \bar{F}_{44}\right] \beta^{2}, \\
& k_{23}=\left(A_{44}-\frac{4}{h^{2}} D_{44}\right) \beta-\frac{4}{3 h^{2}}\left[F_{22} \beta^{3}-\left(F_{21}+2 F_{66}\right) \beta \alpha^{2}\right]-\left(\bar{D}_{44}-\frac{4}{3 h^{2}} \bar{F}_{44}\right) \beta^{3}-\left(\bar{D}_{55}-\frac{4}{3 h^{2}} \bar{F}_{55}\right) \beta \alpha^{2}, \\
& k_{31}=\left[A_{55}-\left(4 / h^{2}\right) D_{55}\right] \alpha, \quad k_{32}=\left[A_{44}-\left(4 / h^{2}\right) D_{44}\right] \beta, \\
& k_{33}=\left[A_{55}-\left(4 / h^{2}\right) D_{55}\right] \alpha^{2}+\left[A_{44}-\left(4 / h^{2}\right) D_{44}\right] \beta^{2}, \\
& m_{11}=I_{3}-\left(4 / 3 h^{2}\right) I_{5}=m_{22}, \quad m_{12}=m_{21}=0, \quad m_{13}=-\left[\bar{I}_{31}+\left(4 / 3 h^{2}\right) I_{5}\right] \alpha, \\
& m_{23}=-\left[\bar{I}_{32}+\left(4 / 3 h^{2}\right) I_{5}\right] \beta, \quad m_{31}=m_{32}=0, \quad m_{33}=I_{1} .
\end{aligned}
$$

Both $[K]$ and $[M]$ are real and nonsymmetric matrices and since $[M]$ is also nonsingular, we can multiply Eq. (14) by $[M]^{-1}$ from the left to obtain, for each $m n$,

$$
[M]^{-1}[K]\{\Delta\}=\omega^{2}[M]^{-1}[M]\{\Delta\}=\omega^{2}[I]\{\Delta\}
$$

by writing $[A]=[M]^{-1}[K]$ the eigenvalue problem is obtained in the form

$$
[A]\{\Delta\}=\omega^{2}[I]\{\Delta\},
$$

where $[A]$ is real and nonsymmetric as well. Consider now the eigenvalue problem associated with $[A]^{T}$. Its eigenvalues $\bar{\omega}^{2}$ are the same as those of $[A]$, so we can write 
$[A]^{T}\{\bar{\Delta}\}=\bar{\omega}^{2}[I]\{\bar{\Delta}\}=\omega^{2}[I]\{\bar{\Delta}\}$.

For this case the biorthogonality condition (see Ref. 11) should be applied:

$\left[\omega_{m n}^{2}-\bar{\omega}_{p q}^{2}\right] \int_{0}^{a} \int_{0}^{b}\{\Delta\}_{m n}\{\bar{\Delta}\}_{p q} d y d x=0$,

where the barred quantities are associated with the eigenvalue problem of the adjoint operator $[A]^{T}$.

The decoupled differential equation for $T_{m n}(t)$ is obtained by using the modal analysis technique:

$\ddot{T}_{m n}(t)+C \dot{T}_{m n}(t)+\omega_{m n}^{2} T_{m n}(t)=\left(1 / J_{m n}\right) F_{m n}(t)$,

where

$$
C=2 \xi_{m n} \omega_{m n}, \quad F_{m n}(t)=\int_{0}^{a} \int_{0}^{b} \bar{W}_{m n} P_{3}(x, y, t) d y d x,
$$

and the norm is

$$
\begin{aligned}
J_{m n}= & \int_{0}^{a} \int_{0}^{b}\left\{I_{1} W_{m n} \bar{W}_{m n}+I_{3}\left(X_{m n} \bar{X}_{m n}+Y_{m n} \bar{Y}_{m n}\right)-\frac{8}{3 h^{2}} I_{5}\left(X_{m n} \bar{X}_{m n}+Y_{m n} \bar{Y}_{m n}+X_{m n} \bar{W}_{m n, x}+Y_{m n} \bar{W}_{m n, y}\right)\right. \\
& \left.-\left(16 / 9 h^{4}\right) I_{7}\left[\left(X_{m n} \bar{X}_{m n}+Y_{m n} \bar{Y}_{m n}+2 X_{m n} \bar{W}_{m n, x}+Y_{m n} \bar{W}_{m n, y}\right)+W_{m n, x} \bar{W}_{m n, x}+W_{m n, y} \bar{W}_{m n, y}\right]\right\} d y d x .
\end{aligned}
$$

The solution of Eq. (20) in the case of homogeneous initial conditions reads

$$
T_{m n}(t)=\frac{1}{J_{m n}} \int_{0}^{t} F_{m n}(\tau) h_{m n}(t-\tau) d \tau
$$

and by the use of Eq. (12), the transverse displacement is expressed as ${ }^{12}$

$$
\begin{aligned}
W(x, y, t) & =\sum_{m, n} \frac{W_{m n}}{J_{m n}} \int_{-\infty}^{t} F_{m n}(\tau) h_{m n}(t-\tau) d \tau \\
& =\sum_{m, n} \frac{W_{m n}}{J_{m n}} \int_{-\infty}^{\infty} \bar{F}_{m n}(W) H_{m n}(W) e^{i \omega \tau} d \omega
\end{aligned}
$$

where

$$
\bar{F}_{m n}(W)=\frac{1}{2 \pi} \int_{-\infty}^{\infty} F_{m n}(t) e^{-i \omega t} d t,
$$

while $H_{m n}(W)$ is the complex frequency response function associated with the $m n$ mode,

$$
H_{m n}(\omega)=\left[1 /\left(\omega_{m n}^{2}-\omega^{2}+i 2 \xi_{m n} \omega_{m n} \omega\right)\right]=L_{m n}^{-1}(\omega) .
$$

\section{RANDOM VIBRATIONS}

The autocorrelation function of the transverse displacement is ${ }^{12}$

$$
R_{W}\left(x_{1}, y_{1}, t_{1} ; x_{2}, y_{2}, t_{2}\right)=E\left[W\left(x_{1}, y_{1}, t_{1}\right) W\left(x_{2}, y_{2}, t_{2}\right)\right] .
$$

For stationary excitation with zero mean we obtain

$$
\begin{aligned}
R_{W}\left(x_{1}, y_{1}, x_{2}, y_{2}, \tau\right)= & \sum_{m, n p, q} \sum_{m n}\left(x_{1}, y_{1}\right) W_{p q}\left(x_{2}, y_{2}\right) \\
& \times \int_{-\infty}^{\infty} S_{Q_{m n} Q_{p q}}(\omega) \\
& \times H_{m n}(\omega) H_{p q}^{*}(\omega) e^{i \omega \tau} d \omega,
\end{aligned}
$$

where

$$
\begin{aligned}
S_{Q_{m n} Q_{p q}}(\omega)= & \frac{1}{J_{m n} J_{p q}} \int_{0}^{a} \int_{0}^{b} \int_{0}^{a} \int_{0}^{b} S_{F}\left(x_{1}, y_{1}, x_{2}, y_{2}, \omega\right) \\
& \times W_{m n}\left(x_{1}, y_{1}\right) W_{p q}\left(x_{2}, y_{2}\right) d y_{2} d x_{2} d y_{1} d x_{1},
\end{aligned}
$$

while

$S_{F}\left(x_{1}, y_{1} ; x_{2}, y_{2}, \omega\right)=\frac{1}{2 \pi} \int_{-\infty}^{\infty} R_{F}\left(x_{1}, y_{1} ; x_{2}, y_{2}, \tau\right) e^{-i \omega \tau} d \tau$

denotes the cross-spectral density function of the applied load. In the following, two cases of random excitation will be considered.

In the case of random excitation the plate is considered to be driven by a point load at $(\bar{x}, \bar{y})$, random in time and characterized by an ideal white-noise correlation function. The counterparts of Eqs. (26) and (27) then read

$$
\begin{aligned}
R_{F}\left(x_{1}, x_{2}, y_{1}, y_{2}, \tau\right)= & R \delta\left(x_{1}-\bar{x}\right) \delta\left(x_{2}-\bar{x}\right) \\
& \times \delta\left(y_{1}-\bar{y}\right) \delta\left(y_{2}-\bar{y}\right) \delta(\tau)
\end{aligned}
$$

and

$$
\begin{aligned}
S_{F}\left(x_{1}, x_{2}, y_{1}, y_{2}, \omega\right)= & \frac{1}{2 \pi} \int_{-\infty}^{\infty} R_{F}\left(x_{1}, x_{2}, y_{1}, y_{2}, \tau\right) e^{-i \omega \tau} d \tau \\
= & (R / 2 \pi) \delta\left(x_{1}-\bar{x}\right) \delta\left(x_{2}-\bar{x}\right) \\
& \times \delta\left(y_{1}-\bar{y}\right) \delta\left(y_{2}-\bar{y}\right) \\
S_{Q_{m n} Q_{p q}}(\omega)= & \frac{1}{J_{m n} J_{p q}} \int_{A_{1}} \int_{A_{2}}\left(\frac{R}{2 \pi} \delta\left(x_{1}-\bar{x}\right) \delta\left(x_{2}-\bar{x}\right)\right. \\
& \times \delta\left(y_{1}-\bar{y}\right) \delta\left(y_{2}-\bar{y}\right) \sin \frac{m \pi}{a} x_{1} \\
& \left.\times \sin \frac{n \pi}{b} y_{1} \sin \frac{p \pi}{a} x_{2} \sin \frac{q \pi}{b} y_{2}\right) d A_{2} d A_{1} \\
= & \frac{1}{J_{m n} J_{p q}} S_{0} \sin \frac{m \pi}{a} \bar{x} \sin \frac{n \pi}{b} \bar{y} \sin \frac{p \pi}{a} \bar{x} \sin \frac{q \pi}{b} \bar{y},
\end{aligned}
$$


where $S_{0}=R / 2 \pi$ and $d A_{i}=d x_{i} d y_{i}(i=1,2)$.

For the case when the load is applied at the center of the plate, i.e., when $\bar{x}=a / 2, \bar{y}=b / 2$, we obtain

$$
\begin{aligned}
S_{Q_{m n} Q_{p q}}(\omega)= & \left(1 / J_{m n} J_{p q}\right) S_{0}\left[(-1)^{(m-1) / 2}(-1)^{(n-1) / 2}\right. \\
& \left.\times(-1)^{(p-1) / 2}(-1)^{(q-1) / 2}\right], \\
& m, n, p, q=1,3,5, \ldots \quad(31)
\end{aligned}
$$

and the mean square of the displacement function at the driven point is

$$
\begin{aligned}
R_{W}\left(\frac{a}{2}, \frac{b}{2}, \frac{a}{2}, \frac{b}{2}, 0\right)= & \sum_{m, n p, q} W_{m n}\left(\frac{a}{b}, \frac{b}{2}\right) W_{p q}\left(\frac{a}{b}, \frac{b}{2}\right) \\
& \times \int_{-\infty}^{\infty} \frac{S_{Q_{m n} Q_{p q}}(\omega)}{L_{m n}(\omega) L_{p q}^{*}(\omega)} d w .
\end{aligned}
$$

The natural frequencies were found to be well separated (see Table I) and for the case of light damping, the autocorrelation terms only are taken into account, ${ }^{12}$ so that

$$
\begin{aligned}
& R_{W}\left(\frac{a}{2}, \frac{b}{2}, \frac{a}{2}, \frac{b}{2}, 0\right) \\
& \quad \simeq S_{0} \sum_{m, n} \frac{1}{J_{m n}^{2}} \sin ^{2} \frac{m \pi}{2} \sin ^{2} \frac{n \pi}{2} \int_{-\infty}^{\infty} \frac{d \omega}{\left|L_{m n}(\omega)\right|^{2}} \\
& \quad=S_{0} \sum_{m, n} \frac{1}{J_{m n}^{2}} \frac{\pi}{2 \xi_{m n} \omega_{m n}^{3}} \quad(m, n=1,3,5, \ldots) .
\end{aligned}
$$

In the second case, the excitation field is due to a turbulent boundary layer pressure fluctuation. Assuming that in the $y$ direction the plate is infinitely long, we obtain the state of cylindrical bending, for which the $2 \times 2$ counterparts of the matrices $[K]$ and $[M]$ are

$$
\begin{aligned}
k_{11}= & {\left[D_{11}-\left(4 / 3 h^{2}\right) F_{11}\right] \alpha^{2}+A_{55}-\left(4 / h^{2}\right) D_{55} } \\
& -\left[\bar{D}_{55}-\left(4 / 3 h^{2}\right) \bar{F}_{55}\right] \alpha^{2}, \\
k_{12}= & {\left[A_{55}-\left(4 / h^{2}\right) D_{55}\right] \alpha-\left(4 / 3 h^{2}\right) F_{11} \alpha^{3} } \\
& -\left[\bar{D}_{55}-\left(4 / 3 h^{2}\right) \bar{F}_{55}\right] \alpha^{3}, \\
k_{22}= & {\left[A_{55}-\left(4 / h^{2}\right) D_{55}\right] \alpha^{2}, } \\
k_{21}= & {\left[A_{55}-\left(4 / h^{2}\right) D_{55}\right] \alpha } \\
m_{11}= & I_{3}-\left(4 / 3 h^{2}\right) I_{5}, \\
m_{12}= & -\left[\bar{I}_{31}+\left(4 / 3 h^{2}\right) I_{5}\right] \alpha \\
m_{22}= & I_{1}, \quad m_{21}=0,
\end{aligned}
$$

TABLE I. Frequencies of cross-ply laminate $\left(0^{\circ}, 90^{\circ}, 90^{\circ}, 0^{\circ}\right), a=b=50 \mathrm{~h}$.

\begin{tabular}{llc}
\hline \hline$m$ & $n$ & Frequencies (rad/s) \\
\hline 1 & 1 & 4859.0675 \\
3 & 1 & 20788.342 \\
5 & 1 & 53872.430 \\
1 & 3 & 33884.710 \\
3 & 3 & 42018.487 \\
5 & 3 & 67897.037 \\
\hline
\end{tabular}

while the associated norm is given by

$$
\begin{aligned}
J_{i}= & \int_{0}^{a}\left(I_{1} W_{i} \bar{W}_{i}+I_{3} X_{i} \bar{X}_{i}-\frac{8}{3 h^{2}} I_{5}\left(X_{i} \bar{X}_{i}+X_{i} \bar{W}_{i, x}\right)\right. \\
& \left.+\frac{16}{9 h^{4}} I_{7}\left(X_{i} \bar{X}_{i}+2 X_{i} \bar{W}_{i, x}+W_{i, x} \bar{W}_{i, x}\right)\right) d x .
\end{aligned}
$$

The mathematical model for the pressure statistics is based on that of Corcos, ${ }^{13}$ with Wilby's approximation ${ }^{14}$

$$
S_{F}(\eta, \omega)=S_{F}(\omega) f_{F}(\eta, \omega) \cos \left(\omega \eta / U_{c}\right),
$$

where

$$
f_{F}(\eta, \omega)=\exp (-\bar{A}|\eta|)=\exp \left[-\left(0.1 \omega / U_{c}\right)|\eta|\right],
$$

$U_{c}$ denotes the mean convection velocity in the flow direction, and $\eta=x_{2}-x_{1}$. The spectral density $S_{F}(\omega)$ is given by $^{15}$

$S_{F}(\omega)= \begin{cases}S_{0}, & \omega \leqslant 1.932\left(0.65 U_{0} / \delta^{*}\right), \\ 2 S_{0} /\left(\omega \delta^{*} / U_{0}\right)^{3}, & \omega>1.932\left(0.65 U_{0} / \delta^{*}\right),\end{cases}$

where

$$
S_{0}=\alpha^{2} 0.75 \times 10^{-5} U_{0}^{3} \delta^{*} \rho_{0}^{2},
$$

$\alpha$ is the fluid dimensionless constant, $\delta^{*}$ denotes the boundary layer displacement thickness, and $\rho_{0}$ is the fluid density.

For the low-frequency range, namely $\omega \delta^{*} / U_{c}<0.2$, we also use Crocker's modification ${ }^{16}$ :

$$
\bar{A}=0.1\left(\omega / U_{c}\right)+0.265 / \delta_{0},
$$

where $\delta_{0}$ is the thickness of the boundary layer.

In order to calculate the mean square of the displacement function, Eq. (26) may equivalently be expressed as ${ }^{12}$

$$
\begin{aligned}
R_{W}\left(\frac{a}{2}, \frac{a}{2}, 0\right)= & \sum_{i} W_{i}^{2}(x) \frac{1}{J_{i}^{2}} \\
& \times \int_{-\infty}^{\infty} \frac{S_{F}(\omega) A_{Q_{i} Q_{i}}(\omega) a^{2}}{\left|L_{i}(\omega)\right|^{2}} d w .
\end{aligned}
$$

Here $A_{Q_{i} Q_{i}}(\omega)$ denotes the joint acceptance, which is evaluated here by using Laplace's asymptotic method. ${ }^{12}$ This yields

$$
\begin{aligned}
A_{Q_{i} Q_{i}}= & \frac{4 d_{i} E_{i}}{\pi i R_{i}}+\frac{32 d_{i} e_{i}}{\pi^{2} i^{2} R_{i}^{2}}\left(E_{i}^{2}-2 e_{i}^{2}\right)(-1)^{i} e^{-A} \sin B_{i} \\
& +\left(8 / \pi^{2} i^{2} R_{i}^{2}\right)\left[E_{i}^{2}-4 e_{i}^{2}\left(1+2 d_{i}^{2}\right)\right] \\
& \times\left[1-(-1)^{i} e^{-A} \cos B_{i}\right]
\end{aligned}
$$

where

hence,

$$
\begin{aligned}
& d_{i}=A / i \pi, \quad e_{i}=B_{i} / i \pi, \quad B_{i}=\left(\omega_{i} / U_{c}\right) a, \quad A=\bar{A} a, \\
& E_{i}=1+d_{i}^{2}+e_{i}^{2}, \quad R_{i}=E_{i}^{2}-4 e_{i}^{2} ;
\end{aligned}
$$

$$
R_{W}\left(\frac{a}{2}, \frac{a}{2}, 0\right)=S_{0} a^{2} \sum_{i} \frac{1}{J_{i}^{2}} A_{Q_{i} Q_{i}} \frac{\pi}{2 \xi_{i} \omega_{i}^{3}}, \quad i=1,3,5, \ldots
$$




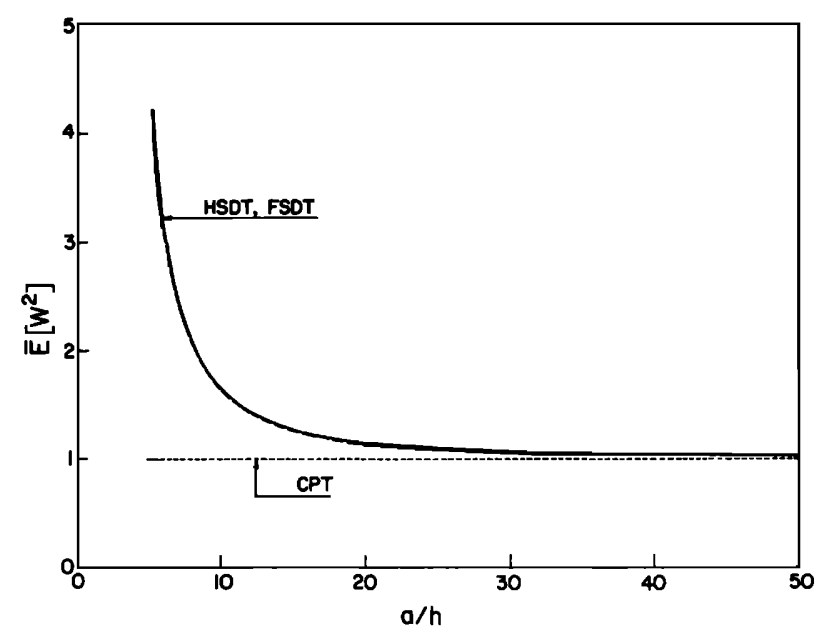

FIG. 1. Relative (to CPT) mean-square transverse displacement versus $a / h$.

\section{NUMERICAL RESULTS AND DISCUSSION}

Two instances will be considered in the numerical illustration. The first is associated with a symmetric cross-ply laminated plate $\left(0^{\circ}, 90^{\circ}, 90^{\circ}, 0^{\circ}\right)$, while the second is associated with a transversely isotropic plate. For the case of the laminated plate, the constituent materials are orthotropic, with the characteristics belonging to a woven graphite fabric and carbon matrix ${ }^{17}$ (material 1 ):

$$
\begin{array}{lll}
E_{1}=25.1 \mathrm{MSI}, & G_{12}=1.36 \mathrm{MSI}, & v_{12}=0.031, \\
E_{2}=4.8 \mathrm{MSI}, & G_{13}=1.2 \mathrm{MSI}, & v_{13}=0.25, \\
E_{3}=0.75 \mathrm{MSI}, & G_{23}=0.47 \mathrm{MSI}, & v_{23}=0.171,
\end{array}
$$

and $\rho=0.075$ PCI.

For the transversely isotropic plate, the material (material 2) is characterized by

$$
\begin{array}{lll}
E=20 \mathrm{MSI}, & v=0.1, & \rho=0.075 \mathrm{PCI}, \\
E^{\prime}=0.75 \mathrm{MSI}, & v^{\prime}=0.25, & G^{\prime}=0.5 \mathrm{MSI},
\end{array}
$$

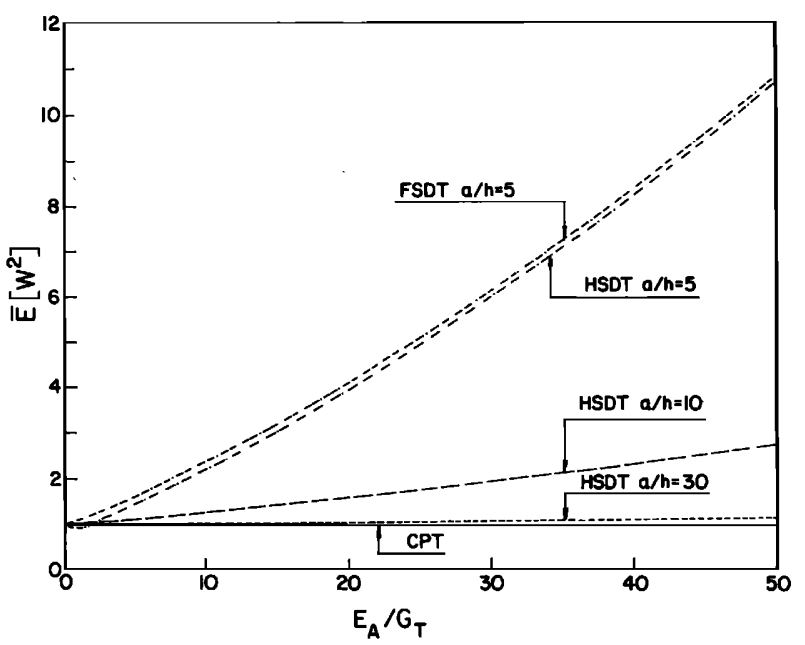

FIG. 2. Relative (to CPT) mean-square transverse displacement versus $E_{A} / G_{T}$

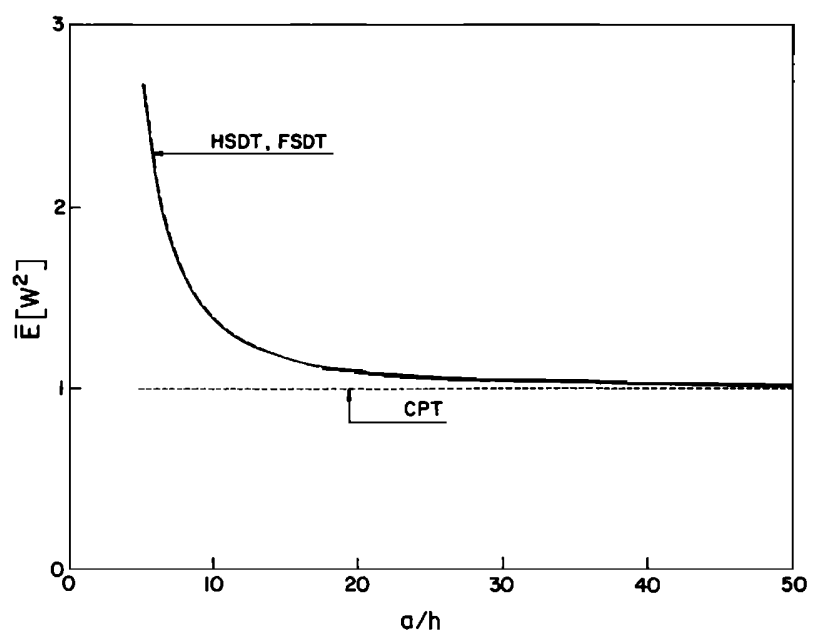

FIG. 3. Relative (to CPT) mean-square transverse displacement versus $a / h$.

where the primed quantities belong to the planes normal to the midplane of the plate (i.e., normal to the plane of isotropy). The turbulent boundary layer pressure field will be modeled by assuming the following properties ${ }^{18}$ :

$$
U_{0}=1888.5 \mathrm{ft} / \mathrm{s}, \quad \delta_{0}=11 \text { in., } \quad \delta^{*}=4.6 \mathrm{in} .
$$

the fluid media is air:

$$
\alpha=3, \quad \rho_{0}=0.00037 \text { slug } / \mathrm{ft}^{3} \text {. }
$$

For comparison, Figs. 1-3 display the results obtained as per the HSDT, FSDT $(k=5 / 6)$, and CPT, where those of the latter are normalized to unity. Figure 1 shows the relative mean square of the transverse displacement for the first load and when material 1 is taken into consideration. Figure 2 exhibits the variation of the relative mean square of the transverse displacement versus $E / G^{\prime}$ ratios for the first load case and where the material of the plate is transversely isotropic (material 2). Figure 3 represents the counterpart of Fig. 1 obtained for the second load case and material 2.

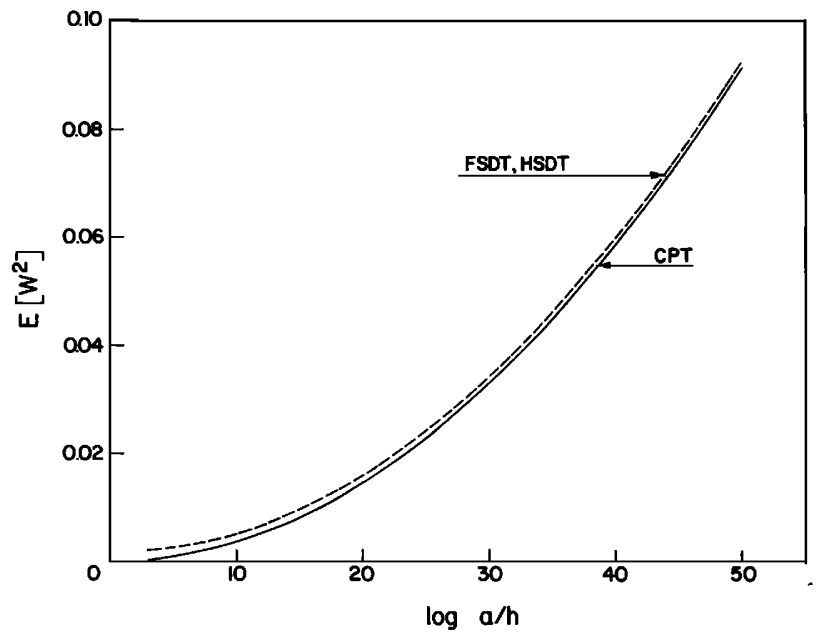

FIG. 4. Variation of the mean-square transverse displacement versus $a / h$. 


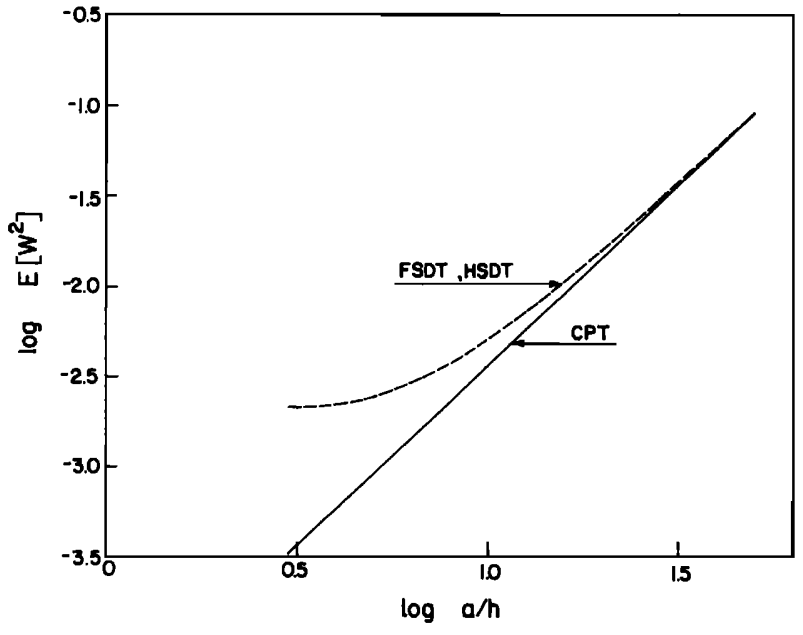

FIG. 5. Variation of the mean-square transverse displacement versus $a / h$ in a logarithmic scale.

Figure 4 shows the variation of the mean-square transverse displacement versus the $a / h$ ratio. Figure 5 shows the same but in a logarithmic scale. It can be seen that the curve associated with the CPT is linear, with a slope of 2 . This indicates that the mean square for this problem can be computed by the relation: mean square $=K a^{2}$, where $K$ is a constant. The curves of the higher-order theories are asymptotic to the CPT. The slope of the CPT line will change as a result of the load distribution function (point, line, or area) and the response problem (static, dynamic, or random vibration).

The numerical results allow us to infer that the transverse shear deformation effect has a great influence on the mean square of the transverse displacement whenever the plate is not a thin one or when the anisotropicity ratios are high enough.

Another conclusion arising from this numerical analysis concerns the similarity of the results provided by the FSDT and HSDT. However, in the present treatment, the need for an arbitrary transverse shear correction factor is eliminated.

\section{ACKNOWLEDGMENTS}

G. Cederbaum gratefully appreciates the financial help provided by the U.S. Army Missile Command's grant of Professor R. A. Heller of the Virginia Polytechnic Institute and State University. Support during the course of this study from the Research Foundation of the Naval Postgraduate School and the Fund of Promotion of Research of the Technion-Israel Institute of Technology for I. Elishakoff is sincerely acknowledged.

'L. Librescu, Elastostatics and Kinematics of Anisotropic and Heterogeneous Shell-Type Structures (Noordhoff, Leiden, The Netherlands, 1975).

${ }^{2} \mathrm{~L}$. Librescu and J. N. Reddy, "A Critical Review and Generalization of Transverse Shear Deformable Anisotropic Plate Theories," in Refined Dynamical Theories of Beams, Plates and Shells and Their Applications, edited by I. Elishakoff and H. Irretier (Springer, Berlin, 1987), pp. 32-43. ${ }^{3}$ S. Thangjithan, L. Librescu, and G. Cederbaum, "Low-Velocity Impact Response of Orthotropic Plates Using a Higher-Order Theory," in AIAA/ ASME/ASCE/AHS 28th Structures, Structural Dynamics and Material Conference, Monterey, CA, 6-8 April 1987, pp. 448-457.

${ }^{4} \mathrm{M}$. Witt and K. Sobczyk, "Dynamic Response of Laminated Plates to Random Loading," Int. J. Solids Structures 16, 231-238 (1980).

${ }^{5} \mathrm{M}$. Witt, "Dynamic Response and Reliability of Laminated Plates to Random Loading," in Refined Dynamical Theories of Beams, Plates and Shells and Their Applications, edited by I. Elishakoff and H. Irretier (Springer, Berlin, 1987), pp. 274-285.

${ }^{6}$ C. Mei and C. B. Prasad, "Effects of Large Deflection and Transverse Shear on Response of Rectangular Symmetric Composite Laminates Subjected to Acoustic Excitation," AIAA Dynamics Specialists Conference, Monterey, CA, 9-10 April 1987, pp. 809-826.

${ }^{7}$ P. C. Yang, C. H. Norris, and Y. Stavsky, "Elastic Wave Propagation in Heterogeneous Plates," Int. J. Solids Structures 2, 665-684 (1965).

${ }^{8} \mathrm{~J}$. M. Whitney and N. J. Pagano, "Shear Deformation in Heterogeneous Anisotropic Plates," J. Appl. Mech. 37, 1031-1036 (1970).

9S. A. Ambartsumian, Theory of Anisotropic Plates, edited by J. E. Ashton (Technomic, Stanford, CT, 1970).

${ }^{10} \mathrm{~J}$. N. Reddy, Energy and Variational Methods in Applied Mechanics (Wiley, New York, 1984).

"L. Meirovitch, Computational Methods in Structural Dynamics (Sijthoff \& Noordhoff, Alphen aan den Rijn, The Netherlands, 1980).

'2I. Elishakoff, Probabilistic Methods in the Theory of Structures (WileyInterscience, New York, 1983).

${ }^{13}$ E. M. Corcos, "Resolution of Pressure in Turbulence," J. Acoust. Soc. Am. 35, 192-199 (1963).

${ }^{14} \mathrm{~J}$. F. Wilby, "Turbulent Boundary Layer Pressure Fluctuations and Their Effect on Adjacent Structures," Jahresbach 1964 Wiss. Ges. Luft-und Raumfahrt.

${ }^{15}$ W. A. Strawderman and R. S. Brand, "Turbulent-Flow-Excited Vibration of a Simply Supported Rectangular Flat Plate," J. Acoust. Soc. Am. 46, 177-192 (1969).

${ }^{16}$ M. S. Crocker, "The Response of a Supersonic Transport Fuselage to Boundary Layer and to Reverberant Noise," J. Sound Vib. 9, 6-20 (1969).

${ }^{17}$ N. J. Pagano and S. R. Soni, "Strength Analysis of Composite Turbine Blades," Proceedings of the First Conference on Composite Materials, Dayton, OH, 7-9 October 1986, pp. 450-471.

${ }^{18}$ Y. K. Lin, S. Maekawa, H. Nijim, and L. Maestrello, "Response of Periodic Beam to Supersonic Boundary-Layer Pressure Fluctuation," in Stochastic Problems in Dynamics, edited by B. L. Clarkson (Pitman, London, 1977), pp. 468-486. 